\title{
Ferramentas Assistivas no Ensino e Aprendizagem de Pessoas com Síndrome de Down: Um Mapeamento Sistemático
}

\author{
Rodrigo Feitosa Gonçalves ${ }^{1}$, Jéssica Farias Cardoso', \\ Rainer Xavier de Amorim ${ }^{1}$ e Odette Mestrinho Passos ${ }^{1}$
}

\author{
${ }^{1}$ Instituto de Ciências Exatas e Tecnologia (ICET). Universidade Federal do \\ Amazonas (UFAM). Caixa Postal 69103-128 - Itacoatiara - AM - Brasil \\ \{feitosar9, jessicafc028\}@gmail.com; rainerxavier, odette\}@ufam.edu.br
}

\begin{abstract}
Teaching children with down syndrome (DS) requires the use of different methods and tools, caused by cognitive, social and language difficulties of these differences. Therefore, this article aims to present the main assistance tools that help in the teaching and learning of people with DS, adopting it, a research methodology based on the principles of Experimental Software Engineering, which it uses when conducting a secondary study: o Systematic Mapping. As a result, 10 assistance tools were identified, the majority of which were contextualized to assist literacy and 3 pedagogical approaches, applied in 30\% of the mapped tools.
\end{abstract}

Resumo. Ensinar crianças com síndrome de down (SD) demanda o uso de métodos e ferramentas diferenciadas, ocasionado por dificuldades cognitivas, social e na linguagem desses indivíduos. Diante disso, este artigo tem como objetivo apresentar as principais ferramentas assistivas que fornecem apoio ao ensino e aprendizagem de pessoas com SD, adotando para isso, uma metodologia de pesquisa fundamentada nos princípios da Engenharia de Software Experimental, que se baseia na condução de um estudo secundário: o Mapeamento Sistemático. Como resultado foram identificas 10 ferramentas assistivas, sendo a maioria contextualizada para o auxilio da alfabetização e 3 abordagens pedagógicas, aplicadas em $30 \%$ das ferramentas mapeadas.

\section{Introdução}

Com o surgimento de softwares voltados para educação pode-se observar inúmeras oportunidades de fomentar e aperfeiçoar o aprendizado de crianças por meio de ferramentas que apresentam conteúdos cada vez mais dinâmicos, com animações e representações gráficas e que representam uma possibilidade de inovação nos recursos. Deste modo, as ferramentas assistivas tem se apresentado como instrumento importante para apoiar as práticas pedagógicas didáticas na sala de aula [Lima, 2013].

Não obstante, na área de educação são indispensáveis tecnologias especiais para estimular adequadamente o ciclo de ensino e aprendizado de pessoas com Necessidades Educativas Especiais (NEE), em específico alunos com Síndrome de Down (SD) [Romero, 2014].

As características da SD se apresentam como uma alteração genética ocorrida durante ou imediatamente após a concepção. A alteração genética se caracteriza pela presença a mais do autossomo 21, ou seja, ao invés do indivíduo apresentar dois 
cromossomos 21, possui três. A esta alteração denomina-se trissomia simples [Schwartzman, 1999].

A SD acarreta na criança gerando um atraso em todas as áreas do desenvolvimento, contudo, não há um padrão pré-definido de desenvolvimento do indivíduo com essa síndrome, de forma que o desenvolvimento cognitivo não está ligado especificamente da alteração cromossômica, mas da influência que a sociedade pode exercer na vida da pessoa com SD [Fernandes, Luciene e Eduardo, 2016]. Assim sendo, atividades educacionais do cotidiano educacional devem ser adaptadas para cada caso, proporcionando o desenvolvimento do indivíduo e aumentando suas capacidades [Ferreira et al., 2018].

Diante do contexto, os aplicativos educacionais, através de smartphones, por exemplo, na sua maioria fornecem atividades lúdicas, com recurso envolvente e colorido, instigando a curiosidade e o aprendizado de maneira divertida, se apresentando como uma alternativa interessante para auxiliar no ensino e aprendizagem de pessoas com SD. Nesse contexto, os aplicativos estimulam o raciocínio e motivam a busca de novos conhecimentos, fornecendo aos alunos um olhar diversificado dos conteúdos didáticos, pois permitem o aprendizado de forma dinâmica [Rodrigues et al., 2015].

Dessa forma, este artigo tem como objetivo apresentar as principais ferramentas assistivas que auxiliam no ensino e aprendizagem de pessoas com SD, com a finalidade de catalogar e identificar as características dessas ferramentas, assim como as abordagens pedagógicas adotadas pelas mesmas.

A metodologia de pesquisa adotada neste trabalho, para coletar as informações de forma a cumprir o objetivo, está fundamentado nos princípios da Engenharia de Software Experimental que se baseia na condução de um estudo secundário: Mapeamento Sistemático (MS). O MS fornece uma visão geral de uma área de pesquisa, identificando a quantidade, os tipos de pesquisas realizadas, os resultados disponíveis, além das frequências de publicações ao longo do tempo para identificar tendências [Petersen et al., 2008].

Kitchenham e Charters (2007), afirmam que estudos de MS têm sido recomendados, sobretudo para áreas de pesquisa onde é difícil visualizar a gama de materiais, relevantes e de alta qualidade, que possam estar disponíveis. Assim sendo, a escolha do MS como proposta para a condução desta pesquisa, justifica-se pelo fato do objetivo da pesquisa ser apenas identificar e utilizar os resultados obtidos para futuras pesquisas.

Como resultado foi possível observar que das 10 ferramentas assistivas, 9 são destinadas para o auxílio da alfabetização inicial, indicando um ponto positivo, pois é uma das fases mais importantes no ensino e aprendizado de pessoas com SD. Com relação as abordagens pedagógicas foram identificadas apenas 3 que foram aplicadas em $30 \%$ das ferramentas.

O restante do artigo está organizado da seguinte maneira: a Seção 2 apresenta a os conceitos relacionados e discute os trabalhos relacionados. A Seção 3 apresenta a metodologia adotada, enquanto a Seção 4 mostra os resultados e as discussões. A Seção 5 apresenta as considerações finais e as propostas de trabalhos futuros. 
IX Congresso Brasileiro de Informática na Educação (CBIE 2020)

Anais do XXXI Simpósio Brasileiro de Informática na Educação (SBIE 2020)

\section{Referencial Teórico}

\subsection{Conceitos Relacionados}

A SD apresenta limitações cognitivas nas crianças, ao nascer, deficiência na memória auditiva, o que dificulta o acompanhamento de instruções faladas e de longa duração, especialmente se elas estiveram recebendo múltiplas informações ou comando de orientações consecutivas. Contudo, está deficiência cognitiva da criança com SD pode ser compensada pela sua habilidade na memória visual, que é mais desenvolvida e apresenta maior capacidade de processamento e assimilação das informações que sua memória auditiva de curto prazo [Lara, Trindade e Nemr, 2007].

Pesquisas tem destacado, que o uso de tecnologia assistiva visa viabilizar ou ampliar habilidades funcionais de pessoas com deficiência e promover uma vida independente e de inclusão. No contexto educacional, a tecnologia assistiva possibilita um aprendizado enriquecido por ferramentas que possibilitam novas oportunidades para que os professores possam explorar e proporcionar de forma dinâmica e lúdica os assuntos pedagógicos em sala de aula, com o uso de computadores que apresentam recursos visuais, sonoros, efeitos e programas computacionais, proporcionando um leque maior de opções para um aprendizado atrativo e motivador [Romero, 2014].

Os motivos do aceitamento da tecnologia assistiva, está ligado a possibilidade de se empregar e desenvolver softwares de forma adequada e de acordo com as necessidades de cada um, a criança com SD consegue progredir de forma satisfatória seu processo cognitivo, visto que o desenvolvimento das pessoas com SD é semelhante ao de pessoas sem a síndrome, as etapas de aprendizado são atingidas, embora em um ritmo mais lento. Além disso, indivíduos com SD desenvolvem diferentes maneiras para manifestar sua capacidade de abstração e simbolização [Pacheco e Oliveira, 2011]

A forma com que as características e sintomas dessa síndrome se apresentam nas pessoas com SD, deixa evidente que há uma grande importância em incentivar indivíduos ao uso de softwares que visem possibilitar maior autonomia e independência das mesmas [Souza et. al., 2017].

\subsection{Trabalhos Relacionados}

O trabalho de Ferreira e Carvalho (2017) teve como objetivo investigar a aplicação de jogos voltados para educação e saúde de crianças com Síndrome de Down, com vistas a retratar o cenário atual e a necessidade de desenvolvimento de ferramentas que possam ser utilizadas como meio de aprimorar a educação inclusiva. Para isso, foi adotada uma revisão de literatura por meio de publicações nas bibliotecas digitais.

Após análise das informações obtidas, os pesquisadores puderam identificar que através do uso de jogos pode-se reduzir o atraso no desenvolvimento de crianças com Síndrome de Down, quando elas são expostas a estímulos adequados de aprendizagem. Esses estímulos podem ser desencadeados com o uso de jogos, quando estes, permitem e exploram estímulos motores, sensoriais e ou cognitivos. Uma das ferramentas destacado no seu estudo foi PlayDown por proporcionar o desenvolvimento de habilidades fundamentais como a memorização, raciocínio lógico, coordenação motora, dentre várias outras habilidades da cognição humana.

O trabalho de Souza e Passos (2019) teve como objetivo analisar tecnologias computacionais que podem ser utilizadas nos diferentes níveis de ensino como auxílio para a melhoria do processo de aprendizagem de alunos da educação especial. Para 
alcançar o objetivo principal, foi realizada um Mapeamento Sistemático da Literatura, por meio de publicações de eventos nacional e internacional.

A partir das análises dos resultados das questões secundárias, os pesquisadores identificaram que as ferramentas tecnológicas expostas nos trabalhos levantados possibilitam o desenvolvimento na educação especial, isso porque as ferramentas identificadas podem ser utilizadas no notebook (software e plataforma web) ou no celular (aplicativo) e podem ser aplicadas em oito tipos de deficiência, gerando facilidade de acesso das ferramentas. Constou-se ainda que as ferramentas apresentam os mais variados assuntos para serem aplicados em sala de aula, como por exemplo, jogos para matemática, português, comunicação, entre outros [Souza e Passos, 2019].

As pesquisas de Ferreira e Carvalho (2017) e Souza e Passos (2019) se assemelham a este trabalho por apresentarem ferramentas e levantamento de trabalhos voltadas para o auxílio de crianças com SD. Entretanto, na pesquisa de Ferreira e Souza (2017), houve apenas o foco no levantamento de trabalhos em duas bibliotecas BIREME e PUBMED, não levando em consideração a avaliação das características dos softwares apresentados. Já o trabalho de Silva e Passos (2019) ficou restrito a uma faixa temporal de buscas de apenas 6 (seis) anos, datados do ano de 2013 a 2018. Outro ponto deste trabalho que vale ressaltar é o fato de o mesmo apresentar apenas buscas em eventos nacionais e internacionais.

O presente trabalho, diferentemente dos acima citados, não possui uma faixa limite de tempo para as buscas realizadas nos simpósios e revistas utilizados para a pesquisa, apresentando buscas nos trabalhos em todos os anos em que os mesmos ocorreram. O mesmo apresenta ainda as características pedagógicas e as diretrizes adotadas no desenvolvimento das ferramentas mapeadas.

\section{Mapeamento Sistemático}

O MS foi baseado no guidelines desenvolvido por Kitchenham e Chartes (2007) e definido em três etapas: (a) Planejamento do Mapeamento: nesse passo, os objetivos da pesquisa são listados e o protocolo do mapeamento é definido; (b) Condução do Mapeamento: durante essa fase, as fontes para o mapeamento são selecionadas, os estudos são identificados, selecionados e avaliados de acordo com os critérios estabelecidos no protocolo do mapeamento e (c) Resultado do Mapeamento: nessa fase, os dados dos estudos são extraídos e sintetizados para serem publicados.

O objetivo deste mapeamento sistemático foi analisar publicações científicas com o propósito de identificar as principais ferramentas assistivas que podem auxiliar no processo de ensino e aprendizagem de pessoas com SD. Sendo assim, este MS buscou respostas para as seguintes questões de pesquisa: [QP1]: Quais as ferramentas assistivas para apoiar o ensino e a aprendizagem de pessoas com SD? e [QP2]: Quais abordagens pedagógicas adotadas para o desenvolvimento das ferramentas assistivas?

Os locais de buscas definidos para a pesquisa foram feitos a partir da busca manual nos anais do (i) Simpósio Brasileiro de Informática na Educação (SBIE), (ii) Workshop de Informática na Escola (WIE), (iii) Conferência Internacional sobre Informática na Educação (TISE) e (iv) Computer on the Beach (COTB) e na Revista (v) Tecnologia da Informação e Comunicação da Faculdade Estácio do Pará (RTICFE-PARÁ), (vi) Novas Tecnologias na Educação (RENOTE) e (vii) Brasileira de Informática na Educação (RBIE). 
A busca foi restringida usando-se palavras-chave específicas para encontrar as publicações de interesse sendo composta a partir da combinação de sinônimos usando os operadores lógicos OR e AND, tanto em português quanto em inglês: ((educação $O U$ ensino OU aprendizagem OU alfabetização OU letramento) OU (ferramenta OU sistema OU aplicação OU software OU ambiente OU jogo)) E (síndrome de down OU distúrbio $O U$ déficit de atenção $O U$ deficiência cognitiva $O U S D$ ).

A pesquisa se restringiu à análise de publicações disponíveis até a data presente da execução do estudo. A seleção das publicações foi realizada em etapas: (1) Busca preliminar das publicações coletadas nas fontes definidas, (2) Análise do título, do resumo e das palavras-chave, (3) Leitura completa e (4) Extração dos dados. Para que a etapa (3) fosse realizada, foi adotado um critério de inclusão, onde a publicação deveria abordar o desenvolvimento de uma ferramenta assistiva para o apoiar o ensino e a aprendizagem de pessoas com SD.

Foram excluídas publicações não relevantes para as questões investigadas e na dúvida quanto à inclusão ou exclusão de algum estudo, o mesmo foi mantido para leitura na íntegra. Tendo como objetivo observar a evolução das pesquisas desenvolvidas na área no decorrer dos anos, este MS não restringiu sua busca a um intervalo específico, entretanto somente publicação em inglês e português foram aceitas.

Na etapa da Condução do MS, a execução ocorreu entre os meses de abril a junho de 2020 e as publicações foram selecionadas de acordo com o critério estabelecido no protocolo. Publicações duplicadas, inacessíveis ou indisponíveis na internet foram descartadas.

De um total de 56 publicações identificadas, após a leitura do título, resumo e palavras-chave, 15 foram selecionadas seguindo o perfil da pesquisa. Todas elas foram lidas na íntegra e ao final 10 publicações foram selecionadas por atenderem o critério de inclusão, apresentados na Tabela 1.

Tabela 1: Estudos primários por base de busca

\begin{tabular}{|c|c|c|c|c|}
\hline Fontes & Ano & Inicialmente & $\begin{array}{c}\text { Resultado da } \\
\text { Etapa 1 }\end{array}$ & $\begin{array}{c}\text { Resultado da } \\
\text { Etapa 2 }\end{array}$ \\
\hline SBIE & $2001-2019$ & 13 & 07 & 6 \\
\hline WIE & $2003-2019$ & 08 & 01 & 0 \\
\hline TISE & $2003-2019$ & 07 & 01 & 0 \\
\hline COTB & $2010-2019$ & 08 & 01 & 1 \\
\hline RTICFE-PARÁ & $2018-2019$ & 05 & 01 & 1 \\
\hline RENOTE & $2003-2019$ & 13 & 02 & 2 \\
\hline RBIE & $1997-2019$ & 02 & 02 & 0 \\
\hline TOTAL & $\mathbf{5 6}$ & $\mathbf{1 5}$ & $\mathbf{1 0}$ \\
\hline
\end{tabular}

\section{Resultados e Discussões}

Com relação à primeira questão de pesquisa "Quais as ferramentas assistivas para apoiar o ensino e a aprendizados de pessoas com SD?" foram identificadas 10 ferramentas conforme pode ser observado na Tabela 2.

Ao analisar a Tabela 2 é possível observar que das 10 ferramentas, 9 (90\%) são destinados ao apoio inicial da alfabetização de pessoas com SD. A publicação [FA8] se destaca por reunir 10 jogos com desafios de diferentes níveis, onde o usuário pode 
IX Congresso Brasileiro de Informática na Educação (CBIE 2020)

Anais do XXXI Simpósio Brasileiro de Informática na Educação (SBIE 2020)

procurar animais escondidos pelo cenário, aprender números, letras e formas geométricas. Além disso, todas as fases são reveladas por um personagem.

Tabela 2. Características das ferramentas assistivas

\begin{tabular}{|c|c|c|c|c|c|}
\hline ID & Título & Objetivo & Faixa Etária & Conteúdo & Evento \\
\hline FA1 & Argot & $\begin{array}{c}\text { Ensino de uma } \\
\text { segunda língua }\end{array}$ & De 10 a 17 anos & Língua Inglesa & SBIE \\
\hline FA2 & $\begin{array}{c}\text { Alfabetizando } \\
\text { Brincando }\end{array}$ & $\begin{array}{c}\text { Auxiliar na } \\
\text { alfabetização }\end{array}$ & NE & Língua Portuguesa & SBIE \\
\hline FA3 & MoviLetrando & $\begin{array}{c}\text { Auxiliar na } \\
\text { alfabetização }\end{array}$ & NE & $\begin{array}{c}\text { Língua Portuguesa e } \\
\text { Matemática }\end{array}$ & SBIE \\
\hline FA4 & ABCÊ Bulir & $\begin{array}{c}\text { Auxiliar na } \\
\text { alfabetização }\end{array}$ & De 6 a 17 anos & Língua Portuguesa & SBIE \\
\hline FA5 & $\begin{array}{c}\text { Plataforma } \\
\text { SAM }\end{array}$ & $\begin{array}{c}\text { Auxiliar na } \\
\text { alfabetização }\end{array}$ & De 13 a 33 anos & Matemática & SBIE \\
\hline FA7 & alfabetizAR & $\begin{array}{c}\text { Auxiliar na } \\
\text { alfabetização }\end{array}$ & De 8 a 46 anos & Língua Portuguesa & SBIE \\
\hline FA8 & PlayDown & $\begin{array}{c}\text { Auxiliar na } \\
\text { alfabetização } \\
\text { alfabetização }\end{array}$ & De 9 a 24 anos & Língua Portuguesa & RENOTE \\
\hline FA9 & $\begin{array}{c}\text { As Aventuras } \\
\text { de Kalebinho }\end{array}$ & $\begin{array}{c}\text { Auxiliar na } \\
\text { alfabetização }\end{array}$ & NE & $\begin{array}{c}\text { Língua Portuguesa e } \\
\text { Matemática }\end{array}$ & RTICFE - PARÁ \\
\hline FA10 & Numicon & $\begin{array}{c}\text { Auxiliar na } \\
\text { alfabetização }\end{array}$ & De 1 a 13 anos & Matemática & COTB \\
\hline
\end{tabular}

Legenda: NE - Não especificado

Em relação a característica "Conteúdo" que se refere ao que tipo de conteúdo que é abordado, é possível verificar que 4 publicações apresentam conteúdo referente a língua portuguesa ([FA2], [FA4], [FA6] e [FA7]). As ferramentas [FA3] e [FA9] abordam o ensino da língua portuguesa e matemática, as ferramentas [FA5] e [FA10] abordam o ensino da matemática, enquanto a ferramenta [FA1] foi a única a apresentar o conteúdo para o ensino da língua inglesa. Essas abordagens de conteúdos se apresentam como uma grande vantagem, pois segundo Bissoto (2018) vemos a oportunidade de se estruturar os sistemas de ensino através de ferramentas para possa atender de forma adequada as pessoas com SD, oportunizando a ela desenvolver novas competências e habilidades.

Uma das informações identificados neste MS foi que $40 \%$ das ferramentas assistivas não especificou a faixa etária do público-alvo, e dentre os que informaram, a idade seria de 1 a 46 anos. Outro ponto importante identificado foi que das 10 ferramentas, $5(50 \%)$ ([FA2], [FA3], [FA7], [FA9] e [FA10]) se caracterizaram para o nível de ensino "Educação Infantill". As ferramentas [FA5], [FA6] e [FA8] se descreveram como apoio ao ensino "Todos os níveis", enquanto as ferramentas [FA1] e [FA4] são para o nível "Ensino Fundamental".

Tendo como objetivo investigar o progresso de pesquisas relacionadas ao desenvolvimento de tecnologias assistivas para apoiar o ensino e aprendizagem de pessoas com SD, foi elaborado um gráfico que apresenta a distribuição das publicações analisadas neste MS agrupadas por ano de publicação (Figura 1). A partir do resultado obtido, constatou-se que apesar da busca não ter sido limitada por um período de tempo, o primeiro estudo publicado nas fontes adotadas, foi em 2007, o que indica que estudos nesta área são relativamente novos.

Entre os anos de 1997 a 2006 não houve nenhuma publicação acerca de tema da pesquisa. Após 9 anos tendo somente dois estudos, no ano de 2017, ocorreu um crescimento no número de publicações relacionadas as ferramentas assistivas para 
pessoas com SD, constatando uma ligeira tendência da comunidade científica em desenvolver pesquisas na área. Vale destacar, que nos anos de 2018 e 2019 houve um decrescimento na pesquisa na área.

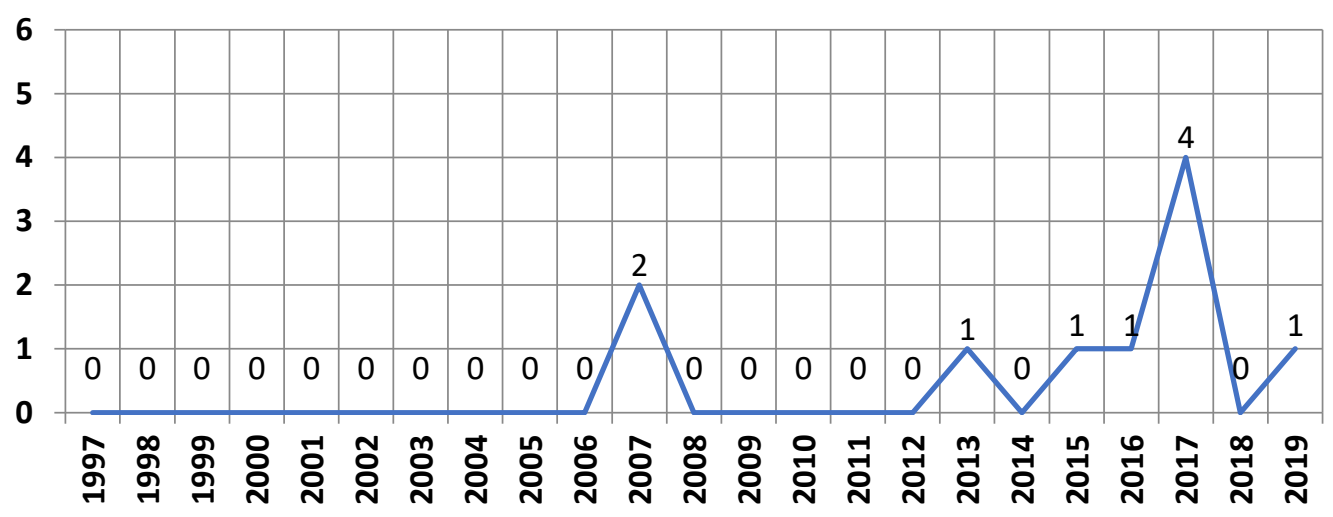

Figura 1. Quantitativo de publicações por ano

A Tabela 3 apresenta as categorias de divisão de cada ferramenta, assim como a Tabela 4 mostra os dispositivos para qual as ferramentas foram desenvolvidas. Em relação as categorias, constatou-se que houve uma alta predominância de jogos, seguido por ferramentas multimídia, que disponibilizam conteúdos instrucionais ilustrativos e atividades didático-pedagógicas como recurso para motivar o aluno através de animações.

Pode-se observar, ainda, que $4(40 \%)$ das ferramentas são aplicativos, ou seja, estão disponíveis para dispositivos móveis, enquanto $4(40 \%)$ são para plataforma desktop. Isso talvez se deva ao fato de que os aplicativos geram facilidades no acesso [Silva, Moura e Soares, 2017]. As ferramentas [FA5] e [FA9] se descrevem como Web.

Tabela 3. Categorias Identificadas

\begin{tabular}{|c|c|}
\hline Categorias & Publicações \\
\hline Ferramenta Multimídia & [FA1] e [FA2] \\
\hline Jogo & [FA3], [FA4], [FA5], [FA6], [FA7], [FA8], [FA9] e [FA10] \\
\hline
\end{tabular}

Tabela 4. Dispositivos

\begin{tabular}{|c|c|}
\hline Dispositivos & Publicações \\
\hline Mobile & {$[$ FA4], [FA6], [FA8] e [FA10] } \\
\hline Desktop & [FA1], [FA2], [FA3] e [FA7] \\
\hline WEB & {$[$ FA5] e [FA9] } \\
\hline
\end{tabular}

Quando se trabalha com o desenvolvimento de ferramentas educacionais é necessário definir uma abordagem para que dê suporte ao aspecto educativo dos mesmos [Abreu et al., 2012]. Melonio e Gennari (2013) destacam a seriedade de adotar parâmetros para se obter softwares mais apropriados ao contexto educacional e com mais qualidade técnica e pedagógica, bem como apontam diretrizes, sob vários aspectos, para a concepção de softwares educacionais.

Dessa forma, com relação à segunda questão de pesquisa "Quais abordagens pedagógicas adotadas para o desenvolvimento das ferramentas assistivas?" foram identificadas 3 abordagens conforme pode ser observado na Tabela 5. Entretanto, é importante salientar, que somente dez estudos $(30 \%)$ adotaram uma abordagem. Esse dado destaca uma carência que pode limitar o potencial educacional das ferramentas desenvolvidas e afetar os benefícios destinados a pessoas com SD. 
Tabela 5. Abordagens pedagógicas

\begin{tabular}{|l|c|}
\hline \multicolumn{1}{|c|}{ Abordagem Pedagógica } & Publicações \\
\hline Global Lecto-Escritura e Decroly & {$[$ FA2] } \\
\hline Storytelling & {$[$ FA9] } \\
\hline Abordagem Multissensorial & [FA10] \\
\hline
\end{tabular}

Estudos como o de Melonio e Gennari (2013) destacam a importância de se adotar parâmetros e métodos para se obter ferramentas de aprendizado mais adequados ao contexto educacional e com mais qualidade técnica e pedagógica, bem como apontam diretrizes, sob vários aspectos, para a concepção de softwares educacionais. Assim, de forma à complementar os resultados, a Tabela 6 mostra as diretrizes que foram adotadas no desenvolvimento das ferramentas assistidas identificadas.

Apesar das ferramentas identificadas apresentarem a mesma finalidade, ou seja, o apoio ao ensino e aprendizagem de pessoas com SD, as diretrizes adotadas em seu desenvolvimento foram diferentes, entretanto, podemos ressaltar que foi dada uma atenção aos aspectos computacionais para $70 \%$ das ferramentas.

\section{Tabela 6. Diretrizes identificadas no desenvolvimento}

\begin{tabular}{|l|c|}
\hline \multicolumn{1}{|c|}{ Diretrizes para Desenvolvimento } & Publicações \\
\hline Interação Humano-Computador (IHC) & {$[$ FA1], [FA2] e [FA8] } \\
\hline Técnicas computacionais para a análise e processamento de imagens & [FA9] e [FA10] \\
\hline Interface natural de usuário & {$[$ FA4] } \\
\hline Técnicas de realidade aumentada & {$[$ FA6] } \\
\hline
\end{tabular}

Outra questão relevante identificada foi se os softwares educacionais identificados apresentam algum instrumento para avaliação e acompanhamento do aprendizado. Neste sentido, apenas as ferramentas [FA4] e [FA5] apresentam mecanismos que possibilitam acompanhar o aluno nas atividades. Entre os instrumentos de avaliação e acompanhamento identificados, o histórico das interações realizadas pelo aluno, foi adotado nos ambientes mobile e web.

A interação do usuário com o computador ocorre por meio de uma interface, onde verificou-se que $70 \%$ das ferramentas utilizaram a tecnologia Interface Gráficas do Usuário (GUI, em inglês, Graphical User Interface), como mostra a Tabela 7, que emprega o método de imagens gráficas e widgets em contexto com o texto para representar as informações e ações disponíveis ao usuário. A GUI é tida como uma interface amigável por proporcionar um conjunto de componentes que possibilitam a utilização da aplicação.

Tabela 7. Tecnologia de interface

\begin{tabular}{|l|c|}
\hline \multicolumn{1}{|c|}{ Tecnologia de Interface } & Publicações \\
\hline Animação 2D & [FA6] \\
\hline Classificador de imagens e GUI & [FA2] \\
\hline GUI & [FA1], [FA4], [FA5], [FA7], [FA8], [FA9] e [F10] \\
\hline Realidade aumentada & [FA3] \\
\hline
\end{tabular}

Quando se trabalha com o desenvolvimento de ferramentas para apoiar o ensino e aprendizado é importante validar o produto não somente no seu desenvolvimento, mas também com seu público alvo, assim obtendo uma ferramenta final capaz de auxiliar o educador e aluno no processo de ensino e aprendizagem. Isso caracteriza a avaliação de software educacional uma etapa indispensável na inserção destas ferramentas em sala de aula [Webber et al., 2009]. 
Com relação ao fato das ferramentas assistivas terem sido ou não validades/testadas, podemos afirmar que das 10, 50\% ([FA1], [FA2], [FA4], [FA5] e [FA9]), foram validadas/testadas especificamente com professores e pessoas com SD e 40\% ([FA6], [FA7], [FA8] e [FA10]) validadas/testadas somente com psicólogos ou profissionais na área da educação. As demais publicações não especificaram se houve ou não a validação com o usuário.

\section{Considerações Finais}

É notável que as tecnologias assistivas se apresentam como instrumento de acessibilidade e inclusão, o qual proporcionam ferramentas ricas que pode auxiliar no desenvolvimento cognitivo das crianças, principalmente as que têm Síndrome de Down. Assim, esse trabalho apresenta um mapeamento sistemático sobre ferramentas assistivas para apoiar o ensino e a aprendizagem de pessoas com Síndrome de Down.

A pesquisa foi realiza em 4 anais de evento nacional/internacional e em 3 revistas científicas. Como principal resultado foram identificadas 10 ferramentas assistivas, sendo a maioria contextualizada para o auxílio da alfabetização. Deste modo, $40 \%$ das ferramentas são aplicativos, $40 \%$ são desktop e $20 \%$ são WEB, ou seja, são voltadas para dispositivos móveis, desktop e WEB, sendo a maioria jogos educacionais, seguido de ferramentas multimidias. Com relação a abordagens pedagógicas foram identificadas apenas 3 que foram aplicadas em 30\% das ferramentas.

Um aspecto relevante foi que $90 \%$ das ferramentas foram testadas com pessoas com SD e com profissionais da área da educação e da saúde. Entretanto, quanto às diretrizes computacionais adotadas em seu desenvolvimento, $70 \%$ das ferramentas receberam uma atenção especial, sendo levado em consideração os aspectos quanto a interface, padrões de projetos e técnicas.

A limitação desse trabalho foi a falta de buscas em bibliotecas digitais, que poderiam acrescentar outros resultados à pesquisa, agregando maior valor $\mathrm{e}$ confiabilidade. Em contra partida, as fontes de revistas e evento nacional e internacional trouxeram resultados satisfatórios e suficientes para responder as questões da pesquisa.

Como trabalhos futuros, espera-se ampliar a pesquisa, por intermédio de buscas em mais fontes e evoluir os questionamentos propostos para que seja possível avaliar quais os métodos de validação mais utilizados nas ferramentas e se os conteúdos propostos foram comparados com métodos tradicionais de ensino. Além disso, desenvolver uma ferramenta que agrega os principais aspectos de relevância para apoiar o ensino e a aprendizagem de pessoas com SD.

\section{Referências}

Abreu, F.; Almeida, A.; Barreiros, E.; Saraiva, J.; Soares, S.; Araújo, A. e Henrique, G. 2012. Métodos, Técnicas e Ferramentas para o Desenvolvimento de Software Educacional: Um Mapeamento Sistemático. XII Simpósio Brasileiro de Informática na Educação (SBIE), Rio de Janeiro, p. 1-10.

Bissoto, M. 2005. O Desenvolvimento Cognitivo e o Processo de Aprendizagem do Portador de Síndrome de Down: Revendo Concepções e Perspectivas Educacionais. Ciências e Cognição, v. 4, n. 2, p. 80-88.

Carvalho, M.; Ferreira, C.; Lobato, P. e Carvalho F. 2017. Ferramentas Aplicadas à Educação e Saúde em Crianças com Síndrome de Down. III Seminário de Tecnologias Aplicadas em Educação e Saúde (STAES), p. 1-10. 
IX Congresso Brasileiro de Informática na Educação (CBIE 2020)

Anais do XXXI Simpósio Brasileiro de Informática na Educação (SBIE 2020)

Fernandes, F.; Luciene, C. e Oliveira, E. 2016. Aplicação de Realidade Aumentada Móvel para Apoio à Alfabetização de Crianças com Autismo. V Anais dos Workshops do Congresso Brasileiro de Informática na Educação (CBIE), p. 1374-1383.

Ferreira, W.; Cordeiro, R.; Aguiar, Y.; Saraiva, J.; Tardif, C. e Galy, E. 2018. Panorama das Publicações Nacionais sobre Autismo, Educação e Tecnologia. Simpósio Brasileiro de Informática na Educação (SBIE), Fortaleza, p. 913-922.

Kitchenham, B. e Charters, S. (2007). Guidelines for Performing Systematic Literature Reviews in Software Engineering. Relatório Técnico Evidence-Based Software Engineering (EBSE), v. 2.3. Disponível em: http://citeseerx.ist.psu.edu/viewdoc/summary?doi=10.1.1.117.471. Acesso em: 15 mar. 2020.

Lara, A.; Trindade, R. e Nemr, K. 2007. Desempenho de Indivíduos com Síndrome de Down nos Testes de Consciência Fonológica Aplicados com e sem Apoio Visual de Figuras. Revista Spech, Language, Hearing Sciences e Education Journal, n. 2, p. 164-73.

Lima, F. Music Spectrum: Imersão Musical para Crianças com Autismo. 2013. 88f. Dissertação (Mestrado em Informática) - Instituto de Computação da Universidade Federal do Amazonas.

Melonio, A. e Gennari, R. , 2007. How to Design Games for Deaf Children: Evidence Based Guidelines. II International Workshop on Evidence-based Technology Enhanced Learning, p. 83-92.

Pacheco, W. e Oliveira, M. 2011. Aprendizagem e Desenvolvimento da Criança com Síndrome de Down: Representações Sociais de Mães e Professoras. Ciência e Cognição, v. 16, n. 3, p. $2-14$.

Petersen, K.; Feldt, R.; Mujtaba, S. e Matisson, M. 2013. Systematic Mapping Studies in Software Engineering. Systematic Mapping Studies in Software Engineering, Bari, p. 1-10.

Rodrigues, M.; Zildomar, F.; José, J. e Isledna, A. 2015. ABCÊ Bulir: Software para o Auxílio no Processo de Alfabetização e Desenvolvimento Cognitivo de Crianças com Síndrome de Down. Simpósio Brasileiro de Informática na Educação (SBIE), Alagoas, p. 867.

Romero, T. Os processos de Ensino e Aprendizagem do Aluno com Sindrome de Down: um Estudo no Noroeste do Paraná. 2014. 32f. Monografia (Especialização em Educação: Métodos e Técnicas de Ensino) - Universidade Tecnológica Federal do Paraná, 2014.

Schwartzman, J. S. Sindrome de Down. São Paulo: Mackenzie, 1999.

Silva, M.; Moura, I. e Soares, A. 2017. Uso de Tecnologias Computacionais para o Ensino de Crianças com Transtorno do Espectro Autista: Um Mapeamento Sistemático da Literatura. XXVIII Simpósio Brasileiro de Informática na Educação (SBIE), Recife, p. 173182.

Souza, A. e Passos O. 2019. Educação Especial: Um Mapeamento Sistemático sobre Tecnologias Computacionais. 2019. 66f. Monografia (Graduação em Sistema de Informação). Universidade Federal Amazonas, Itacoatiara.

Souza, D.; Trindade, G.; Fernandes e Bonifácio, B. 2017. AlfabetizAR: Uma Aplicação Móvel com base na Realidade Aumentada como Ferramenta de Apoio no Processo de Alfabetização de Portadores de Sindrome de Down. XXVIII Simpósio Brasileiro de Informática na Educação (SBIE), Recife, p. 897-905.

Webber, C.; Boff, E. e Bono, F. 2009. Ferramenta Especialista para Avaliação de Software Educacional. XX Simpósio Brasileiro de Informática na Educação (SBIE), Florianópolis, p. $1-10$.

Werner, H 2009. O Processo da Construção do Número, o Lúdico e TICs como Recursos Metodológicos para a Criança com Deficiência Intelectual. Caderno (Pedagógico apresentado à Coordenação do Programa de Desenvolvimento Educacional) - Programa de Desenvolvimento Educacional (PDE). 